\title{
A multistage successive approximation method for Riccati differential equations
}

\author{
Petrus Setyo Prabowo', Sudi Mungkasi² \\ ${ }^{1}$ Department of Electrical Engineering, Faculty of Science and Technology, Sanata Dharma University, Mrican, Tromol \\ Pos 29, Yogyakarta 55002, Indonesia \\ ${ }^{2}$ Department of Mathematics, Faculty of Science and Technology, Sanata Dharma University, Mrican, Tromol Pos 29, \\ Yogyakarta 55002, Indonesia
}

\begin{tabular}{l}
\hline \hline Article Info \\
\hline Article history: \\
Received Aug 9, 2020 \\
Revised Nov 3, 2020 \\
Accepted Apr 6, 2021 \\
\end{tabular}

Keywords:

Multistage method Piecewise method Riccati differential equations Successive approximation Variational iteration method

\begin{abstract}
Riccati differential equations have played important roles in the theory and practice of control systems engineering. Our goal in this paper is to propose a new multistage successive approximation method for solving Riccati differential equations. The multistage successive approximation method is derived from an existing piecewise variational iteration method for solving Riccati differential equations. The multistage successive approximation method is simpler in terms of computing implementation in comparison with the existing piecewise variational iteration method. Computational tests show that the order of accuracy of the multistage successive approximation method can be made higher by simply taking more number of successive iterations in the multistage evolution. Furthermore, taking small size of each subinterval and taking large number of iterations in the multistage evolution lead that our proposed method produces small error and becomes high order accurate.
\end{abstract}

This is an open access article under the CC BY-SA license.

\section{Corresponding Author:}

Sudi Mungkasi

Department of Mathematics

Faculty of Science and Technology

Sanata Dharma University, Yogyakarta, Indonesia

Email: sudi@usd.ac.id

\section{INTRODUCTION}

Mathematical modelling and simulation have been applied extensively in the areas of telecommunication [1], [2], computing [3]-[5], electronics [6], [7] and control [8], [9]. One of important models in these areas is the Riccati differential equation. Riccati differential equations occur in control systems engineering. Control systems engineering itself has played important roles in electrical engineering and related areas [10]. Riccati differential equations are quadratic with respect to the unknown function. These equations are named after a Venetian mathematician, Jacopo Francesco Riccati (1676-1754) [11]. The term Riccati equation is also used to refer to an analogous matrix equation occuring in quadratic control problems. The non-dynamic steady-state version of them is referred to as the algebraic Riccati equation.

Riccati equations and their properties are applied in recent publications [12]-[15]. A number of studies relating to Riccati equations are also reported in the literature [16]-[21]. Due to the importance of Riccati equations, we focus on proposing a computing method for solving Riccati differential equations.

Amongst available methods in the literature, successive approximation and variational iteration methods are able to provide accurate solutions near the initial point of the domain. Successive approximation methods are successful in solving various problems [22]-[23]. Variational iteration methods are also powerful in solving a wide variety of mathematical models [24]-[27]. Interestingly, these two methods (successive 
approximation and variational iteration methods) are identical for a particular setting, as reported by Jafari [28].

Our contribution (our goal) in this paper is to propose a new multistage successive approximation method for solving Riccati differential equations. We recall an existing piecewise variational iteration method due to Geng, et al. [29]. We modify the method of Geng, et al. [29] to obtain the multistage successive approximation method. The modification leads to a simpler implementation of the resulting method in terms of computation. Riccati differential equations have the following form

$$
\frac{d y}{d x}=p(x)+q(x) y(x)+r(x) y^{2}(x)
$$

on $0<x \leq X$, with initial condition

$$
y(0)=y_{0}
$$

where $x$ is the free variable, $y(x)$ is the unknown function dependent on $x, X$ is a known positive constant, and $y_{0}$ is a given constant. We note that if $p(x)=0$, (1) becomes a Bernoulli equation. If $r(x)=0$, (1) reduces to a first order linear ordinary differential equation. The Riccati differential equation with the case of $p(x)=0$ or $r(x)=0$ can be solved using standard methods for ordinary differential equations. In this paper, we consider that $p(x) \neq 0$ and $r(x) \neq 0$. Due to the important roles of Riccati differential equations in control systems engineering, a simple but accurate solver is desired. Providing a simple and accurate method for solving Riccati differential equations is the aim of this paper.

This paper is organised as follows. We explain the problem that we want to tackle in section 2. We propose a multistage successive approximation method for solving Riccati differential equations in section 3 . Results and discussion are provided in section 4 . The paper is concluded in section 5.

\section{PROBLEM DESCRIPTION}

In this section, we recall an existing piecewise variational iteration method for solving Riccati differential equations due to Geng, et al. [29]. The variational iteration method itself, for the general case, was originally proposed by $\mathrm{He}[30]-[32]$.

Considering Riccati differential (1) with initial condition (2) on domain $0 \leq x \leq X$, Geng, et al. [29] took the correction functional

$$
y_{n+1}(x)=y_{n}(x)+\int_{0}^{x} \lambda(\xi)\left[\frac{d y_{n}(\xi)}{d \xi}-q(\xi) \bar{y}_{n}(\xi)-r(\xi) \bar{y}_{n}^{2}(\xi)-p(\xi)\right] d \xi,
$$

where $\bar{y}_{n}$ is a restricted variation, that is, $\delta \bar{y}_{n}=0 ; \lambda(\xi)$ is a Lagrange multiplier, which should be determined optimally. Taking the variation of (3), we obtain

$$
\delta y_{n+1}(x)=\delta y_{n}(x)+\delta \int_{0}^{x} \lambda(\xi)\left[\frac{d y_{n}(\xi)}{d \xi}-q(\xi) \bar{y}_{n}(\xi)-r(\xi) \bar{y}_{n}^{2}(\xi)-p(\xi)\right] d \xi,
$$

Simplifying (4), we obtain

$$
\delta y_{n+1}(x)=\delta y_{n}(x)+\delta \int_{0}^{x} \lambda(\xi)\left[\frac{d y_{n}(\xi)}{d \xi}\right] d \xi,
$$

Integrating (5) by parts, we have

$$
\delta y_{n+1}(x)=\delta\left[(1+\lambda(x)) y_{n}(x)\right]+\delta \int_{0}^{x} y_{n}(\xi) \lambda^{\prime}(\xi) d \xi,
$$

Considering (6), we come to the following stationary conditions

$$
1+\lambda(x)=0, \lambda^{\prime}(\xi)=0 .
$$

Solving stationary conditions (7), we obtain that the optimal Lagrange multiplier is

$$
\lambda(\xi)=-1 .
$$


Therefore, with Lagrange multiplier (8), the variational iteration method due to Geng, et al. [29] is

$$
y_{n+1}(x)=y_{n}(x)-\int_{0}^{x}\left[\frac{d y_{n}(\xi)}{d \xi}-q(\xi) y_{n}(\xi)-r(\xi) y_{n}^{2}(\xi)-p(\xi)\right] d \xi,
$$

which is for solving Riccati differential (1) with initial condition (2) on domain $0 \leq x \leq X$.

Realising that the variational iteration method (9) produces analytical approximate solutions which are accurate only for points close enough to the initial position, Geng, et al. [29] implemented the method (9) piecewisely. By piecewisely, Geng, et al. [29] meant that the original interval $I=[0, X]$ was subdivided into a finite number of subintervals $I_{j}=\left[x_{j-1}, x_{j}\right]$, where $j=1,2,3, \ldots, J$ for a positive integer $J$. The width of each subinterval is assumed to be the same, that is, $\Delta x=x_{j}-x_{j-1}$ for all $j$. With this setting, we have $J+1$ discrete points of the original interval $I=[0, X]$, that is, $x_{0}=0, x_{1}=\Delta x, x_{2}=2 \Delta x, \ldots, x_{j}=J \Delta x=X$. We denote $y_{j, n}(x)$ the analytical approximate solution on the $j$ th subinterval at the $n$th variational iteration. Suppose that the maximum number of variational iterations is $N$, where $N$ is a specified positive integer. This means that $n=0,1,2, \ldots, N$.

The piecewise variational iteration method due to Geng, et al. [29] works as follows. As the first step, on subinterval $I_{1}=\left[x_{0}, x_{1}\right]$, we take the initialization

$$
y_{1,0}(x)=y\left(x_{0}\right)=y(0)=y_{0}
$$

and iterations

$$
y_{1, n+1}(x)=y_{1, n}(x)-\int_{x_{0}}^{x}\left[\frac{d y_{1, n}(\xi)}{d \xi}-q(\xi) y_{1, n}(\xi)-r(\xi) y_{1, n}^{2}(\xi)-p(\xi)\right] d \xi
$$

for $n=0,1,2, \ldots, N-1$. As shown in (10) and (11), as the second step, on subinterval $I_{2}=\left[x_{1}, x_{2}\right]$, we take the initialization

$$
y_{2,0}(x)=y_{1, N}\left(x_{1}\right)
$$

and iterations

$$
y_{2, n+1}(x)=y_{2, n}(x)-\int_{x_{1}}^{x}\left[\frac{d y_{2, n}(\xi)}{d \xi}-q(\xi) y_{2, n}(\xi)-r(\xi) y_{2, n}^{2}(\xi)-p(\xi)\right] d \xi,
$$

for $n=0,1,2, \ldots, N-1$. As shown in (12) and (13), as the next steps, on subintervals $I_{j}=\left[x_{j-1}, x_{j}\right]$, where $j=3,4,5, \ldots, J$, we take the initialization

$$
y_{j, 0}(x)=y_{j-1, N}\left(x_{j-1}\right)
$$

and iterations

$$
y_{j, n+1}(x)=y_{j, n}(x)-\int_{x_{j-1}}^{x}\left[\frac{d y_{j, n}(\xi)}{d \xi}-q(\xi) y_{j, n}(\xi)-r(\xi) y_{j, n}^{2}(\xi)-p(\xi)\right] d \xi
$$

for $n=0,1,2, \ldots, N-1$. Using (10)-(15), we obtain the solution on the whole domain.

Now, the most expensive computation with the formulation of Geng, et al. [29] above lies on the part where we need to calculate the derivative $d y_{j, n}(\xi) / d \xi$ and integrate the results from $x_{j-1}$ to $x \in I_{j}$. These tasks are redundant. To obtain a simpler method for solving Riccati differential equations, we need to modify this piecewise variational iteration method of Geng, et al. [29]. This is the problem that we aim to solve in this paper.

\section{PROPOSED MULTISTAGE SUCCESSIVE APPROXIMATION METHOD}

In this section, we propose a modification of the piecewise variational iteration method of Geng, et al. [29], so that the modified method is simpler in terms of computing implementation, yet its accuracy does not change. We reconsider the iterative formula of the variational iteration method (9) and do the integration for the term having the derivative of the unknown function, so as shown in (9) becomes 


$$
y_{n+1}(x)=y_{0}+\int_{0}^{x}\left[q(\xi) y_{n}(\xi)+r(\xi) y_{n}^{2}(\xi)+p(\xi)\right] d \xi
$$

where for $n=0,1,2, \ldots, N-1$. Iterative formula (16) is Picard's successive approximation method for Riccati differential (1). The iterative in (16) of the successive approximation method is equivalent to the iterative formula of the variational iteration method (9), but in (16) is simpler.

Now, we shall implement the iterative (16) of the successive approximation method (SAM) piecewisely. The resulting method is called multistage successive approximation method (MSAM) for solving Riccati differential equations. Our MSAM works as follows. As the first step, on subinterval $I_{1}=$ $\left[x_{0} ; x_{1}\right]$, we take the initialization

$$
y_{1,0}(x)=y\left(x_{0}\right)=y(0)=y_{0}
$$

and iterations

$$
y_{1, n+1}(x)=y_{0}+\int_{x_{0}}^{x}\left[q(\xi) y_{1, n}(\xi)+r(\xi) y_{1, n}^{2}(\xi)+p(\xi)\right] d \xi
$$

for $n=0,1,2, \ldots, N-1$. As shown in (17) and (18), as the second step, on subinterval $I_{2}=\left[x_{1}, x_{2}\right]$, we take the initialization

$$
y_{2,0}(x)=y_{1, N}\left(x_{1}\right)
$$

and iterations

$$
y_{2, n+1}(x)=y_{2,0}(x)+\int_{x_{1}}^{x}\left[q(\xi) y_{2, n}(\xi)+r(\xi) y_{2, n}^{2}(\xi)+p(\xi)\right] d \xi
$$

for $n=0,1,2, \ldots, N-1$. As shown in (19) and (20), as the second step, on subinterval $I_{j}=\left[x_{j-1}, x_{j}\right]$, we take the initialization

$$
y_{j, 0}(x)=y_{j-1, N}\left(x_{j-1}\right)
$$

and iterations

$$
y_{j, n+1}(x)=y_{j, 0}(x)+\int_{x_{j-1}}^{x}\left[q(\xi) y_{j, n}(\xi)+r(\xi) y_{j, n}^{2}(\xi)+p(\xi)\right] d \xi,
$$

for $n=0,1,2, \ldots, N-1$. Using (17)-(22), we obtain the solution on the whole domain.

To optimise our MSAM further, in the computer implementation, we compute the successive approximation formula symbolically only once:

$$
y_{j, n+1}(x)=y_{j-1, N}\left(x_{j-1}\right)+\int_{x_{j-1}}^{x}\left[q(\xi) y_{j, n}(\xi)+r(\xi) y_{j, n}^{2}(\xi)+p(\xi)\right] d \xi,
$$

for $n=0,1,2, \ldots, N-1$. Then, the obtained symbolic formula from (23) is used to solve the Riccati differential equation on each subinterval $I_{j}$ consecutively for $j=1,2,3, \ldots, J$. Here, we speficy that

$$
y_{0, n}\left(x_{0}\right)=y_{0}
$$

for all $n$. In (24) means that the given initial value is used as the starting point of solution.

\section{RESULTS AND DISCUSSION}

In this section, we provide research results on the performance tests of our proposed method and discuss about them. We take two computational tests, namely, a Riccati differential equation with constant coefficients and a Riccati differential equation involving a variable coefficient. Error on the considered domain is defined as the average of relative errors at all discrete points on the domain.

\subsection{Riccati differential equation with constant coefficients}

As the first test, we consider the Riccati differential equation with constant coefficients [29]: 


$$
\frac{d y(x)}{d x}=1+2 y(x)-y^{2}(x), 0<x \leq 4,
$$

having initial condition

$$
y(0)=0
$$

The exact solution to this problem is

$$
y(x)=1+\sqrt{2} \tanh \left(\sqrt{2} x+\frac{1}{2} \log \left(\frac{-1+\sqrt{2}}{1+\sqrt{2}}\right)\right) .
$$

Our computational experiments show that the standard SAM is not able to solve the problem on thewhole domain. In contrast, our proposed MSAM is able to solve the problem on the whole domain accurately. These phenomena are shown in Figure 1(a), where SAM and MSAM use 3 successive iterations, and in addition, for MSAM we use $\Delta x=0.1$.

To investigate further about the performance of MSAM, we record the errors and their orders of convergence in Tables 1-4. We obtain that the number of successive iterations in the MSAM evolution determines the order of convergence of the solution. One successive iteration in the MSAM evolution leads that MSAM is a first order method. This is observed as $\Delta x$ approaches to zero, the order of convergence tends to one, as recorded in Table 1. Two successive iterations in the MSAM evolution leads that MSAM is a second order method, because as $\Delta x$ approaches to zero, the order of convergence tends to two, as recorded in Table 2. Three successive iterations in the MSAM evolution leads that MSAM is a third order method, because as $\Delta x$ approaches to zero, the order of convergence tends to three, as recorded in Table 3. Similarly, four successive iterations in the MSAM evolution leads that MSAM is a fourth order method, because as $\Delta x$ approaches to zero, the order of convergence tends to four, as recorded in Table 4. In general, smaller $\Delta x$ results in smaller error. Furthermore, more successive iterations results in higher order accurate method.

Table 1. Error and order of convergence of MSAM solution for Riccati differential equation with constant coefficients, in which we use 1 iteration in the MSAM evolution. Error is computed on interval $[0,4]$

\begin{tabular}{ccc}
\hline$\Delta x$ & Average of Relative Error & Order of Convergence \\
\hline 0.25 & $4.420 \mathrm{E}-02$ & - \\
0.125 & $2.425 \mathrm{E}-02$ & 0.87 \\
0.0625 & $1.274 \mathrm{E}-02$ & 0.93 \\
0.03125 & $6.536 \mathrm{E}-03$ & 0.96 \\
0.015625 & $3.311 \mathrm{E}-03$ & 0.98 \\
\hline
\end{tabular}

Table 2. Error and order of convergence of MSAM solution for Riccati differential equation with constant coefficients, in which we use 2 iterations in the MSAM evolution. Error is computed on interval $[0,4]$

\begin{tabular}{ccc}
\hline$\Delta x$ & Average of Relative Error & Order of Convergence \\
\hline 0.25 & $7.393 \mathrm{E}-03$ & - \\
0.125 & $2.022 \mathrm{E}-03$ & 1.87 \\
0.0625 & $5.395 \mathrm{E}-04$ & 1.91 \\
0.03125 & $1.399 \mathrm{E}-04$ & 1.95 \\
0.015625 & $3.565 \mathrm{E}-05$ & 1.97 \\
\hline
\end{tabular}

Table 3. Error and order of convergence of MSAM solution for Riccati differential equation with constant coefficients, in which we use 3 iterations in the MSAM evolution. Error is computed on interval $[0,4]$

\begin{tabular}{ccc}
\hline$\Delta x$ & Average of Relative Error & Order of Convergence \\
\hline 0.25 & $6.674 \mathrm{E}-04$ & - \\
0.125 & $9.271 \mathrm{E}-05$ & 2.85 \\
0.0625 & $1.252 \mathrm{E}-05$ & 2.89 \\
0.03125 & $1.636 \mathrm{E}-06$ & 2.94 \\
0.015625 & $2.094 \mathrm{E}-07$ & 2.97 \\
\hline
\end{tabular}

Table 4. Error and order of convergence of MSAM solution for Riccati differential equation with constant coefficients, in which we use 4 iterations in the MSAM evolution. Error is computed on interval $[0,4]$

\begin{tabular}{ccc}
\hline$\Delta x$ & Average of Relative Error & Order of Convergence \\
\hline 0.25 & $7.349 \mathrm{E}-05$ & - \\
0.125 & $5.049 \mathrm{E}-06$ & 3.86 \\
0.0625 & $3.388 \mathrm{E}-07$ & 3.90 \\
0.03125 & $2.205 \mathrm{E}-08$ & 3.94 \\
0.015625 & $1.407 \mathrm{E}-09$ & 3.97 \\
\hline
\end{tabular}




\subsection{Riccati differential equation involving a variable coefficient}

As the second test, let us consider the Riccati differential equation with a variable coefficient [29]:

$$
\frac{d y(x)}{d x}=1+x^{2}-y^{2}(x), 0<x \leq 4,
$$

with initial condition

$$
y(0)=1
$$

The exact solution to this problem is

$$
y(x)=x+\frac{e^{-x^{2}}}{1+\int_{0}^{x} e^{-\xi^{2} d \xi}}
$$

For this second test, the standard SAM is not able to solve the problem, even for the first half $[0,2]$ of the given domain $[0,4]$, as shown in Figure 1(b). In contrast, our proposed MSAM is able to solve the problem on the whole domain [0,4] accurately. For this Figure 1(b), SAM and MSAM use 3 successive iterations, and in addition, for MSAM we take $\Delta x=0.1$.

To investigate further about the performance of MSAM in solving this second test case, we record the errors and their orders of convergence in Tables 5-8. The behaviour of MSAM in this test case is consistent with that of MSAM in the previous test case. We obtain that the number of successive iterations in the MSAM evolution determines the order of convergence of the solution. One successive iteration in the MSAM evolution leads that MSAM is a first order method. This is observed as $\Delta x$ approaches to zero, the order of convergence tends to one, as recorded in Table 5. Two successive iterations in the MSAM evolution leads that MSAM is a second order method. This is observed as $\Delta x$ approaches to zero, the order of convergence tends to two, as recorded in Table 6. Three successive iterations in the MSAM evolution leads that MSAM is a third order method. This is observed as $\Delta x$ approaches to zero, the order of convergence tends to three, as recorded in Table 7. Similarly, four successive iterations in the MSAM evolution leads that MSAM is a fourth order method, because as $\Delta x$ approaches to zero, the order of convergence tends to four, as recorded in Table 8. Again, in general, smaller $\Delta x$ results in a more accurate method. Furthermore, more number of successive iterations makes MSAM to be higher order accurate.

With our accurate results in this paper, we are confident that MSAM is a reliable method to be used for other kinds of initial value problems. The idea of MSAM could be adapted for continuous versions of discrete problems in computing [33], and it could be extended to be analysed using advanced mathematical tools, such as, algebraic geometry and conformal mapping.

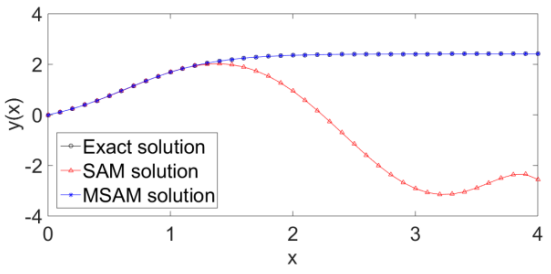

(a)

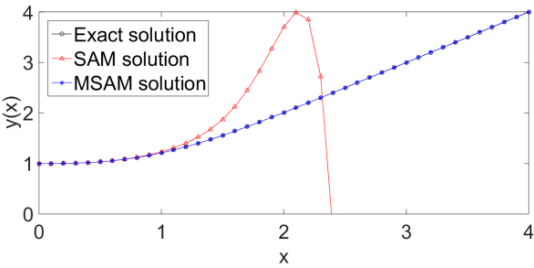

(b)

Figure 1. Exact, SAM, and MSAM solutions on interval [0, 4]. SAM solution is accurate only at points close to the initial condition. MSAM solution coincides graphically with the exact solution, (a) Results of the first test case, (b) Results of the second test case

Table 5. Error and order of convergence of MSAM solution for Riccati differential equation involving a variable coefficient, in which we use 1 iteration in the MSAM evolution on interval $[0,4]$

\begin{tabular}{ccc}
\hline$\Delta x$ & Average of Relative Error & Order of Convergence \\
\hline 0.25 & $3.544 \mathrm{E}-02$ & - \\
0.125 & $1.764 \mathrm{E}-02$ & 1.01 \\
0.0625 & $8.802 \mathrm{E}-03$ & 1.00 \\
0.03125 & $4.396 \mathrm{E}-03$ & 1.00 \\
0.015625 & $2.197 \mathrm{E}-03$ & 1.00 \\
\hline
\end{tabular}


Table 6. Error and order of convergence of MSAM solution for Riccati differential equation involving a variable coefficient, in which we use 2 iterations in the MSAM evolution on interval $[0,4]$

\begin{tabular}{ccc}
\hline$\Delta x$ & Average of Relative Error & Order of Convergence \\
\hline 0.25 & $3.408 \mathrm{E}-02$ & - \\
0.125 & $4.625 \mathrm{E}-03$ & 2.88 \\
0.0625 & $9.436 \mathrm{E}-04$ & 2.29 \\
0.03125 & $2.165 \mathrm{E}-04$ & 2.12 \\
0.015625 & $5.202 \mathrm{E}-05$ & 2.06 \\
\hline
\end{tabular}

Table 7. Error and order of convergence of MSAM solution for Riccati differential equation involving a variable coefficient, in which we use 3 iterations in the MSAM evolution on interval $[0,4]$

\begin{tabular}{ccc}
\hline$\Delta x$ & Average of Relative Error & Order of Convergence \\
\hline 0.25 & $6.406 \mathrm{E}-03$ & - \\
0.125 & $6.894 \mathrm{E}-04$ & 3.22 \\
0.0625 & $7.439 \mathrm{E}-05$ & 3.21 \\
0.03125 & $8.555 \mathrm{E}-06$ & 3.12 \\
0.015625 & $1.024 \mathrm{E}-06$ & 3.06 \\
\hline
\end{tabular}

Table 8. Error and order of convergence of MSAM solution for Riccati differential equation involving a variable coefficient, in which we use 4 iterations in the MSAM evolution on interval $[0,4]$

\begin{tabular}{ccc}
\hline$\Delta x$ & Average of Relative Error & Order of Convergence \\
\hline 0.25 & $2.393 \mathrm{E}-03$ & - \\
0.125 & $1.019 \mathrm{E}-04$ & 4.55 \\
0.0625 & $5.294 \mathrm{E}-06$ & 4.27 \\
0.03125 & $3.007 \mathrm{E}-07$ & 4.14 \\
0.015625 & $1.790 \mathrm{E}-08$ & 4.07 \\
\hline
\end{tabular}

\section{CONCLUSION}

We have proposed successfully a new multistage successive approximation method for solving Riccati differential equations. The main advantage of the proposed method is that it is simpler than the existing variational iteration method for solving Riccati differential equations. The proposed method is analytically equivalent to the existing method, but simpler in terms of computing implementation. We have tested the performance of the multistage successive approximation method in cases that their exact solutions are known. The order of accuracy of the proposed method can be made higher by simply taking more number of successive iterations in the multistage evolution. Obviously, taking smaller size of each subinterval and taking more number of iterations in the multistage evolution lead that our proposed method produces smaller error and it becomes higher order accurate. With these results, in cases that the exact solutions to Riccati differential equations are not known, we are confident to propose the use of the multistage successive approximation method for solving them to obtain accurate approximate solutions.

\section{ACKNOWLEDGEMENTS}

The work of Petrus Setyo Prabowo was financially supported by Sanata Dharma University. The work of Sudi Mungkasi was financially supported by the Ministry of Research and Technology/National Agency for Research and Innovation (RISTEK-BRIN) of the Republic of Indonesia in the form of a research grant of Penelitian Terapan Unggulan Perguruan Tinggi (PTUPT) scheme year 2021 under announcement number B/112/E3/RA.00/2021. These financial supports are gratefully acknowledged by the authors.

\section{REFERENCES}

[1] O. Faruqe, R. Akhter and M. T. Amin, "A low power wideband varactorless VCO using tunable active inductor," TELKOMNIKA Telecommunication Computing Electronics Control, vol. 18, no. 1, pp. 264-271, 2020, doi: 10.12928/telkomnika.v18i1.11365.

[2] M. Takbiri, H. Zarei and A. Bijari, "A new CMOS fully differential low noise amplifier for wideband applications," TELKOMNIKA Telecommunication Computing Electronics Control, vol. 16, no. 3, pp. 1083-1091, 2018, doi: 10.12928/TELKOMNIKA.v16i3.7630.

[3] J. Shukla, A. Joshi and R. Tyagi, "PAPR analysis of OFDM system using AI based multiple signal representation methods," TELKOMNIKA Telecommunication Computing Electronics Control, vol. 17, no. 6,pp. 2983-2991, 2019, doi: 10.12928/telkomnika.v17i6.11511. 
[4] F. Laouafi, A. Boukadoum and S. Leulmi, "A hybrid formulation between differential evolution and simulated annealing algorithms for optimal reactive power dispatch," TELKOMNIKA Telecommunication Computing Electronics Control, vol. 16, no. 2, pp. 513-524, 2018, doi: 10.12928/telkomnika.v16i3.8434.

[5] H. Wang and C. Song, "An improved adaptive niche differential evolution algorithm," TELKOMNIKA Telecommunication Computing Electronics Control, vol. 14, no. 3, pp. 1035-1041, 2016, doi: 10.12928/telkomnika.v14i3.3591.

[6] Z. Abidin, E. Maulana, R. K. Subroto and Wijono, "Transistor mismatch effect on common-mode gain of crosscoupled amplifier," Telkomnika, vol. 17, no. 1, pp. 448-452, 2019, doi: 10.12928/TELKOMNIKA.v17i1.11587.

[7] Tjendro and S. Mungkasi, "Formal expansion method for solving an electrical circuit model," TELKOMNIKA Telecommunication Computing Electronics Control, vol. 17, no. 3, pp. 1338-1343, 2019, doi: 10.12928/telkomnika.v17i3.10318.

[8] I. Parkhomey, J. Boiko, N. Tsopa, I. Zeniv and O. Eromenko, "Assessment of quality indicators of the automatic control system influence of accident interference," TELKOMNIKA Telecommunication Computing Electronics Control, vol. 18, no. 4, pp. 2070-2079, 2020, doi: 10.12928/TELKOMNIKA.v18i4.15601.

[9] K. V. Santhosh and B. R. Navada, "An adaptive liquid level controller using multi sensor data fusion," TELKOMNIKA Telecommunication Computing Electronics Control, vol. 16, no. 5, pp. 2465-2473, 2018, doi: 10.12928/telkomnika.v16i5.9074.

[10] F. E. Hoyos, J. E. Candelo, and J. A. Taborda, "Selection and validation of mathematical models of power converters using rapid modeling and control prototyping methods," International Journal of Electrical and Computer Engineering (IJECE), vol. 8, no. 3, pp. 1551-1568, 2018, doi: 10.11591/ijece.v8i3.pp1551-1568.

[11] S. Bittanti, "Count Riccati and the early days of the Riccati equation," In: S. Bittanti, A. J. Laub and J. C. Willems (eds) The Riccati Equation, Communications and Control Engineering Series, Berlin: Springer, 1991.

[12] H. R. Nohooji, I. Howard and L. Cui, "Optimal robot-environment interaction using inverse differential Riccati equation," Asian Journal of Control, vol. 22, no. 4, pp. 1401-1410, 2020, doi: 10.1002/asjc.2066.

[13] L. Zhang, H. Y. Fan and E. K. W. Chu "Krylov subspace methods for discrete-time algebraic Riccati equations," Applied Numerical Mathematics, vol. 152, pp. 499-510, 2020, doi: 10.1016/j.apnum.2019.11.006.

[14] L. Zhang, H. Y. Fan and E. K. W. Chu, "Inheritance properties of Krylov subspace methods for continuous-time algebraic Riccati equations," Journal of Computational and Applied Mathematics, vol. 371, p. 112685, 2020, doi: 10.1016/j.cam.2019.112685.

[15] J. Liu, L. Wang and Y. Bai, "New estimates of upper bounds for the solutions of the continuous algebraic Riccati equation and the redundant control inputs problems," Automatica, vol. 116, p. 108936, 2020, doi: 10.1016/j.automatica.2020.108936.

[16] T. Song and B. Liu, "Solvability and optimal stabilization controls of discrete-time mean-field stochastic system with infinite horizon," Advances in Difference Equations, vol. 2020, no. 1, p. 187, 2020, doi: 10.1186/s13662-02002639-4.

[17] Y. Chai, J. Luo, N. Han and J. Xie, "Linear quadratic differential game approach for attitude takeover control of failed spacecraft," Acta Astronautica, vol. 175, pp. 142-154, 2020, doi: 10.1016/j.actaastro.2020.04.023.

[18] J. Moon, "Linear-quadratic mean field stochastic zero-sum differential games," Automatica, vol. 120, p. 109067, 2020, doi: 10.1016/j.automatica.2020.109067.

[19] A. Khamis and D. Zydek, "Finite horizon optimal nonlinear spacecraft attitude control," Journal of the Astronautical Sciences, vol. 67, no. 3, pp. 1002-1020, 2020, doi: 10.1007/s40295-019-00189-w.

[20] J. Dannatt, I. R. Petersen and A. Lanzon, "Strictly negative imaginary state feedback control with a prescribed degree of stability," Automatica, vol. 119, p. 109079, 2020, doi: 10.1016/j.automatica.2020.109079.

[21] A. Feddaoui, N. Boizot, E. Busvelle and V. Hugel, "High-gain extended Kalman filter for continuous-discrete systems with asynchronous measurements," International Journal of Control, vol. 93, no. 8, pp. 2001-2014, 2020, doi: 10.1080/00207179.2018.1539525.

[22] M. A. Jankowska, "On elastoplastic analysis of some plane stress problems with meshless methods and successive approximations method," Engineering Analysis with Boundary Elements, vol. 95, pp. 12-24, 2018, doi: 10.1016/j.enganabound.2018.06.013.

[23] S. Salahshour, A. Ahmadian and C. S. Chan, "Successive approximation method for Caputo q-fractional IVPs," Communications in Nonlinear Science and Numerical Simulation, vol. 24, no. 1-3, pp. 153-158, 2015, doi: 10.1016/j.cnsns.2014.12.014.

[24] B. Zhu, Q. Xu, M. Li and Y. Li, "Nonlinear free and forced vibrations of porous functionally graded pipes conveying fluid and resting on nonlinear elastic foundation," Composite Structures, vol. 252, p. 112672, 2020, doi: 10.1016/j.compstruct.2020.112672.

[25] Attaullah and M. Sohaib, "Mathematical modeling and numerical simulation of HIV infection model," Results in Applied Mathematics, vol. 7, p. 100118, 2020, doi: 10.1016/j.rinam.2020.100118.

[26] K. Al-Khaled and A. Hazaimeh, "Comparison methods for solving non-linear Sturm-Liouville eigenvalues problems," Symmetry, vol. 12, no. 7, p. 1179, 2020, doi: 10.3390/sym12071179.

[27] P. L. Ndlovu and R. J. Moitsheki, "Steady state heat transfer analysis in a rectangular moving porous fin," Propulsion and Power Research, vol. 9, no. 2, pp. 188-196, 2020, doi: 10.1016/j.jppr.2020.03.002.

[28] H. Jafari, "A comparison between the variational iteration method and the successive approximations method," Applied Mathematics Letters, vol. 32, no. 1, pp. 1-5, 2014, doi: 10.1016/j.aml.2014.02.004. 
[29] F. Geng, Y. Lin and M. Cui, "A piecewise variational iteration method for Riccati differential equations," Computers and Mathematics with Applications, vol. 58, no. 11-12, pp. 2518-2522, 2009, doi: 10.1016/j.camwa.2009.03.063.

[30] J. H. He, "Variational iteration method for delay differential equations," Communications in Nonlinear Science and Numerical Simulation, vol. 2, no. 4, pp. 235-236, 1997, doi: 10.1016/S1007-5704(97)90008-3.

[31] J. H. He, "Variational iteration method - A kind of non-linear analytical technique: Some examples," International Journal of Non-Linear Mechanics, vol. 34, no. 4, pp. 699-708, 1999, doi: 10.1016/S0020-7462(98)00048-1.

[32] J. H. He, "Variational iteration method for autonomous ordinary differential systems," Applied Mathematics and Computation, vol. 114, no. 2-3, pp. 115-123, 2000, doi: 10.1016/S0096-3003(99)00104-6.

[33] S. Parikh, D. Dave, R. Patel and N. Doshi, "Security and privacy issues in cloud, fog and edge computing," Procedia Computer Science, vol. 160, pp. 734-739, 2019, doi: 10.1016/j.procs.2019.11.018.

\section{BIOGRAPHIES OF AUTHORS}

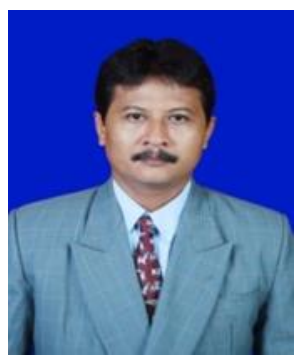

Petrus Setyo Prabowo is an assistant professor at the Department of Electrical Engineering, Faculty of Science and Technology, Sanata Dharma University, Yogyakarta, Indonesia. He obtained the bachelor's degree Sarjana Teknik in Electrical Engineering from Gadjah Mada University, Yogyakarta, Indonesia in 1997. He received the master's degree Magister Teknik in Electrical Engineering from the National Institute of Science and Technology (Institut Sains dan Teknologi Nasional), Jakarta, Indonesia in 2010. He has been an Intermediate Professional Engineer (Insinyur Profesional Madya) according to the Institution of Engineers Indonesia (Persatuan Insinyur Indonesia) since 2016. His research interests include control and optimization in electric power systems. He was the Head of the Department of Electrical Engineering, Faculty of Science and Technology, Sanata Dharma University in 2012-2020.

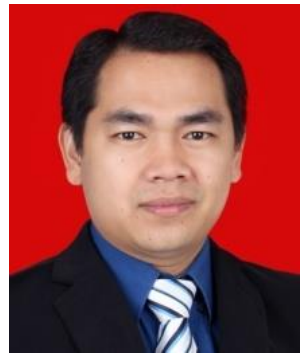

Sudi Mungkasi has been with the Department of Mathematics, Faculty of Science and Technology, Sanata Dharma University, Yogyakarta, Indonesia since 2005. He is currently an associate professor at the Department of Mathematics and the Dean of the Faculty of Science and Technology at Sanata Dharma University. He received the bachelor's degree in mathematics from Gadjah Mada University, Yogyakarta, Indonesia in 2004. Both master's and PhD degrees in mathematical sciences were obtained from The Australian National University, Canberra, Australia in 2008 and 2013, respectively. He was a postdoctoral fellow at the Mathematical Sciences Institute, The Australian National University in 2013. His research interests include applied and computational mathematics for physical, biological, chemical, and engineering problems, such as, electrical engineering problems. 